\title{
Introduction: Why There Should Be a Cognitive Anthropology of Science*
}

\author{
Christophe HeINTZ**
}

\begin{abstract}
I argue that questions, methods and theories drawn from cognitive anthropology are particularly appropriate for the study of science. I also emphasize the role of cognitive anthropology of science for the integration of cognitive and social studies of science. Finally, I briefly introduce the papers and attempt to draw the main directions of research.
\end{abstract}

\section{What's in the label: Cognitive anthropological approach in science studies}

The cognitive anthropology of science is at the crossroads of several rapidly developing disciplines: cognitive science, which increasingly provides tools for the study of scientific thinking; science studies, and in particular the anthropology of science, which is enriching the subject with numerous case studies; naturalised epistemology, which is constantly reworking its philosophical assumptions thus opening new directions for the naturalist study of science; and finally, cognitive anthropology and ethnoscience, which make a valuable contribution in terms of theory, methods and empirical data.

Thus, the cognitive anthropology of science benefits from several paradigms, traditions and research methods. First, cognitive anthropologists can

\footnotetext{
*Acknowledgement: I thank Dan Sperber for his help and advice on the editing of this volume. The work was supported by a stipend from the Max Planck Society; I especially thank Lorraine Daston for welcoming me in her department, at the Max Planck Institute for the History of Science.

**Institut Jean Nicod - EHESS (Paris) \& Max Planck Institute for the History of Science (Berlin).
} 
show how cognitive constraints have contributed, together with historical and cultural factors, to the contents of a given science. Second, sciences can be analysed as specific cultural models or schemas that frame individuals' cognition. Scientists at work, and, more controversially, people in their everyday activities, appeal to specific ways of thinking informed by the 'culture of science.' Third, sciences are cultural objects of particular relevance for the cross-cultural study of notions such as truth or causality, and cognitive operations such as reasoning or categorising. Fourth, scientific practice can be analysed as cognition distributed among scientists and scientific instruments.

And yet, the project remains controversial. This is so because of its explicit objective to combine and integrate the explanatory power of the cognitive and social sciences.

First, cognitive anthropological approaches to science assume that science is a natural phenomenon. This naturalistic credo faces resistance from the renewed assertion of the special status of science as a rational enterprise (e.g. Laudan, 1977, some protagonists of the Science War). From this, some have concluded that only cognitive studies can reveal the real nature of Science, that scientific discovery by computer was a refutation of the strong programme in the sociology of scientific knowledge (Slezak, 1989), and some have emphasised that "cognitive theories do not agree with the relativist epistemology advocated within the sociology of knowledge" (Freedman, 1997; see also the dismissive attitude of Carruthers et al., 2003). ${ }^{1}$ But while these reactions have mostly targeted the social studies of science, cognitive studies have not been spared from doubt and criticism. From within cognitive science, Fodor (1983) takes the example of scientific cognition in order to advance his "First Law of the Nonexistence

\footnotetext{
${ }^{1}$ In their 'very short recent history' of the philosophy of science, Carruthers \& al. dedicate a section to 'Science and the Social,' which they introduce by "our story so far has mostly been of good news - with philosophy of science in the last century, like science itself, arguably progressing and/or getting somewhat closer to the truth. But one outgrowth of the historical turn in philosophy of science was a form of social constructivism or relativism about science" (Bloor, 1976; Rorty, 1979; Latour and Woolgar, 1986; Shapin, 1994). "[...] social constructivism has not found wide acceptance among philosophers of science generally." In his contribution to the volume Carruthers mention again the "social constructivist' account of science, of the sort that Gopnik and Melzoff find so rebarbative (and rightly so, in my view)."
} 
of Cognitive Science": scientific cognition, according to Fodor, is just too complex to be accounted for. From the social studies of science, the doubts and criticisms have been represented by the notorious call for a moratorium on cognitive studies of science (Latour \& Woolgar, 1986: 280; Latour, 1987: 247). Lastly, the project of integrating social and cognitive studies has met much resistance, mainly because it goes against the "Standard Social Science Model" and toward a "Causal Integrated Model" (Tooby \& Cosmides, 1992). Cognitive anthropologists know well the oppositions that their project raises. Regarding the study of science, the same divide between cognitive and social studies occurred. It is not just that there is no collaborative work, but that some authors emphasize the presumed incompatibility of the approaches. Thus, Fuller \& al. (1989) present their edited book, with the otherwise promising title 'The Cognitive Turn: Sociological and Psychological Perspectives on Science' (1989) with: "our historians, philosophers, sociologists, and psychologists [...] have drawn new lines of battle." What a scientific achievement!

If we were to combine these points of views, a cognitive anthropology of science would have everything wrong with it: the cognitive, the anthropological, and its putting them together... And yet, the above doubts and dismissive attitudes bear an anti-scientific attitude that is at odds with the naturalistic spirit that science studies claims to hold. The failure of the foundationalist projects in the philosophy of science induces at least some modesty vis-à-vis one's own approach and theories of science. But this modesty is absent in the dismissal of the others' scientific projects, when justified by the claim to maintain a distance vis-à-vis the whole of science. $^{2}$ On the other hand, naturalism requires an optimistic faith in the development of science that is absent from Fodor's argument or from arguments that exclude scientific practice from scientific investigation because of its 'rational' character. Rather, one should investigate science with the scientific theories, tools and methods at hand, which is what cognitive anthropology does by combining the explanatory power of the social and cognitive sciences. Moreover, cognitive anthropology of science actually

\footnotetext{
${ }^{2}$ This claim which is apparent in e.g. Woolgar (1989) and which is denounced as foundationalist by Nickles (1989: 226). It is also present in the claim to characterise scientific cognition in terms of necessary and sufficient conditions.
} 
provides a rich soil for solving many of the problems alluded to by the critics. Here is why:

'Cognitive' as a naturalised notion

Nersessian (in Press) asserts that one can find a cause of Latour \& Woolgar's moratorium in the partially justified association of an erroneous Cartesianism with cognitive studies of science. The criticism, she continues, would then bear on a cognitive reductivism in which "the social and the cultural environments [...] are treated as abstract content on which cognitive processes operate." Yet, such a justified criticism would not, by itself, lead to a moratorium and a massive rejection of cognitive research on science. The association that led to the moratorium was moreover between logical positivism and cognitive studies. This association was rendered possible because 'cognitive' is, unfortunately, often used interchangeably with 'rational' (see, e.g. Longino, 2002). Cognitive students of science could therefore be seen as merely transferring the positivists' foundational logic and its purported virtue to lead to the truth within the heads of the scientists. ${ }^{3}$ Anthropology has long been struggling with these issues. Since Levy Bruhl's distinction between primitive "pre-logical" thought and rational modern thought, Rationality has often been challenged as a mere Western ethnocentric presupposition (Bryan Wilson, Ed. Rationality). Cognitive anthropology allows restating the problem anew by analysing cognition not as thought processes leading to true beliefs (in normal conditions) but as mental mechanisms or properties sustaining the many diverse cultures. In that perspective, cognitive anthropology of science will not aim at discovering the essence of science; it will rather investigate the ways in which the mental apparatus allows the production of the cultural phenomena found in the history of science, and reciprocally how the specific cultural environments of science constrain or inform mental processes.

\footnotetext{
${ }^{3}$ Woolgar characterizes the cognitivist stance as "the idea that mental or other inner processes enable the rightful perception of an already existing world" (1989:206; my emphasis). It is of course a caricature of the stance and Woolgar attacks a straw man: illusions are well studied in cognitive psychology. Yet, subtler versions of that stance can indeed be found in cognitive studies of science: see e.g. Pickering's (1991) criticism of Giere (1988); Alvin Goldman (1986, 1994) asserts likewise that some cognitive processes reliably lead to (scientific) truth.
} 
The interplay between scientific cognition and scientific cultures

With a cognitive anthropological approach, therefore, one can investigate empirically the characteristics of scientific thoughts and practices. Being freed from a normative agenda or its associated essentialist claims, one can aim at a description of scientific cultures - and even, at pointing out the most salient traits. But if one can identify scientific communities, if only as self proclaimed communities that work at specifying their identities and maintaining cultural boundaries through differentiation (Ellen, this volume), it is far more difficult to characterise these cultures in terms of cognitive practices. Again, the question is a classic one in cognitive anthropology: do different cultures imply different ways of thinking? The most radical answer can be found in strong cognitive relativism, ${ }^{4}$ according to which culture does have a very important impact in framing mental processes and abilities. The Sapir-Worf hypothesis, for instance, hypothesises that thinking and perceiving radically differ from one language community to another. Most recent findings, however, have shown that the mind is richly endowed with innate structures and abilities that allow and strongly constrain human thinking. Berlin and Kay's study (1969), for instance, suggests that the extensions of colour terms are constrained by universal cognitive and biological factors rather than being totally relative to cultural contingencies that would constrain perception. There is, in cognitive anthropology, a research trend that aims at articulating the causal relation from the innate cognitive constraints of our mental apparatus to the diversity of cultural phenomena (e.g. Barkow, Cosmides \& Tooby, 1992; Hirschfeld \& Gelman, 1994; Sperber, 1996; Sperber \& Hirschfeld, 2004). Applying this research programme to the sciences of life led Atran (1990) to hypothesize the existence of an innate ability to reason about living kinds that has constrained the history of Natural History as well as current research practices in neo-darwinist theory.

\footnotetext{
"'Cognitive relativism,' which essentially relates to the question of the plasticity of the mind and the extent in which it is framed by culture, should not be conflated with 'epistemological relativism,' which asserts that truth itself is relative to individuals or communities or with 'methodological epistemic relativism' which prescribes to bracket the truth of a belief in order to study the causes of the associated truth claims. The first is an empirical question, the second a metaphysical position, and the third a methodological prescription (which was originally stated as the 'symetry principle,' Bloor, 1991 [1976]).
} 
It thus appears that scientific theories are largely unintuitive: They are generating cognitive conflict with antagonistic cognitive dispositions and representations promoted by other cultural domains (see Poling \& Evans, this volume, for the example of neo-darwinism with regard to basic intuitions and religious beliefs). Yet, even if scientific knowledge is not a direct product of our minds, but is the fruit of a long and complicated historical and cultural development, it is still assumed that scientists are human beings with the same endowed biological mental apparatus as lay people and their hunter-gatherer ancestors.

\section{The continuity hypothesis}

The above assumption implies the following alternative: either the mind/ brain is sufficiently plastic to allow drastic changes in the organisation of the mental apparatus during the course of scientific education (Churchland, 1988), or lay and scientific mental processes remain mainly similar - this is the continuity hypothesis. The question provides a central research direction in cognitive anthropology of science and sends us back to much other research: Neurobiology on the plasticity of the brain, developmental psychology on the impact of scientific education, evolutionary psychology with regard to the plausibility of the abilities hypothesized, comparative and historical anthropology, etc. In cognitive anthropology of science the issues concern the relationships between folk theories and scientific knowledge and practices. Do cognitive dispositions afford and constrain science in the same way as they afford and constrain folk knowledge? Can the development of science be seen as a cultural process of emancipation from cognitive constraints? Does science manage cognitive resources such as memory, imagination and reasoning abilities in the same way as other cultural institutions such as religion? The study of apprenticeship and enculturation during scientific education allow us to understand how thought processes are oriented, framed or made possible so as to engender specifically scientific thinking (Roth; Alac \& Hutchins; Poling

\& Evans and Kurz-Milcke et al., this volume). The problem of continuity between lay and scientific cognition, between children's and scientists' thinking, is also a way of exploiting and specifying analogies and identities among cognitive phenomena. There are, consequently, many ways to understand the continuity hypothesis, depending on which 
cognitive phenomena are taken to be stable across cultures and life span, or which analogy one wants to draw (see Carruthers, 2002 and Erana \& Martinez, this volume). In this respect, two cognitive phenomena are put at the center of the research stage: mental models and situated cognition.

\section{Mental models}

Mental models are mental constructs that represent a situation, event or process and have structural similarities with what they represent JohnsonLaird, 1983). They are studied both in cognitive studies of science and in cognitive anthropology, but with a rather different perspective. In cognitive studies of science, the research on mental models was initiated as a reaction to the shortcomings of logical positivism, which aimed at describing scientific knowledge in terms of axiomatic systems, and scientific reasoning in terms of logical, syntactic, operations on propositional representations. Contrary to this view, mental models have been shown to play a major role in the 'cognitive structure of scientific theories' (Giere, 1988, 1994) and in scientific reasoning - such as analogical, visual and simulative modelling (e.g. Nersessian, 1992; Magnani \& Nersessian, 2002). In cognitive anthropology, the emphasis has been on cultural models, which are mental models that are culturally shared, such as the American model of marriage (Quinn, 1987). But in science also, a mental model acquires a significant role only when it is shared by a scientific community. In mathematics, for instance, proof methods, argumentative methods and proof strategies (Van Bendegem \& Van Kerkhove, this volume) appear to be cultural models for mathematicians in much the same way as Quinn's model of marriage for Americans. Yet, scientific cultural models will often appear highly complex and unintuitive, often requiring the reorganisation of our understanding of a domain through conceptual change. This raises again the problem of scientific education, communication, and questions on the possibility and processes of conceptual change (Carey \& Spelke, 1994). While the importance of the distribution of a scientific mental model among the scientific community shows well the usefulness of the cognitive anthropological approach, there is also another sense in which scientific models are culturally shared and consisting of social phenomena. Scientific models, indeed, do not only take place in scientists' minds: they also often have an institutional, material and social reality (see Ellen, $\S 7$ and Kurz- 
Milcke et al., this volume). This is because of the embodied, situated and distributed aspects of (scientific) cognition.

Embodied, situated and distributed scientific cognition

Mainly coming from cognitive anthropology this time (e.g. Loan, 1988; Hutchins, 1995), but also from an environmental perspective in cognitive psychology, philosophy of mind and education (e.g. the work of $\mathrm{L}$. Barsalou, A. Clark, J. Greeno), cognitive studies of science show today a significant interest in a notion of cognition that seriously takes into consideration the non-mental aspects of cognition (see Nersessian, in press, $\S 3$, for a review). At the forefront of the research is the role of visual representations (Spranzi, Gooding; Roth and Alac \& Hutchins, this volume), which again demonstrates the limits of the traditional propositional account of scientific cognition. As when one adds large numbers, laying their material representations on the paper and then adding each row following a detailed procedure (McClelland \& al., 1986: 44-48), scientists appear to rely largely on visual representations, as well as on other external representations, for knowledge production and communication. Artefacts also have a full role in the generation of scientific representations (Gooding; Tweney; Kurz-Milcke et al. and Erana \& Martinez). In the end, when the flow and transformations of scientific representations are studied without an arbitrary restriction to purely mental processes, one can take on the task of describing cognitive systems that are distributed among human agents and artefacts. The ensuing framework leads to the analysis of the social organisation of the cognitive systems that produce scientific knowledge, and points out the essential phenomena 'where the cognitive and the social merge' (Giere \& Moffat, 2003). What are the specific cognitive architectures of scientific institutions? How do these structures relate to the production of scientific knowledge? (See Kurz-Milcke et al., Erana \& Martinez, and Giere, this volume for explicit treatment of these questions).

On the determinations of scientific cognition

Identifying scientific cultures is often easy, because of their self proclaimed constitution. But determining and describing their salient social and cognitive traits is something much harder that requires detailed empirical 
investigation. In a naturalistic perspective, however, I take it that the final goal is to account for scientific knowledge production with a description of the causal chains that determine and eventually produce Science - a cultural and cognitive object. I hope that the above paragraphs have hinted at the fact that these causal chains actually include both cognitive (including mental) events and social, cultural and historical events (this dichotomy merging at many places). Cognitive anthropology, as the study of thought in cultural context, but also as the study of culture as constituted through people's thinking and interacting, should therefore provide a rich medium for the study of science. Rather than combining the problems mentioned above, a cognitive anthropological approach solves them by stressing the social or the cognitive when needed - i.e. when the determinants or causal factors of the scientific event to be explained are actually (but non exclusively) social or cognitive.

Take Fodor's argument: he points out that in scientific reasoning, anything can be made relevant to one's topic, and any proposition can enter one's reasoning. This, he maintains, renders the cognitive processes involved untraceable. Spranzi's (this volume) case study is an example of such reasoning where an analogy is drawn between two distinct phenomena: Galileo interprets the black marks on the moon as similar to the shadows thrown by mountains on the earth. Now, Spranzi argues, the analogy did not pop up out of the blue - which would have exemplified a mysterious 'Fodorian' (isotropic) cognitive event. She shows, on the contrary, that it was rendered possible through a historical process of bootstrapping. In other words, the cultural context made some ideas and representations available to Galileo, thus framing his cognitive environment (Sperber \& Wilson, 1986, § 1.8) and making the analogy possible. We therefore have a case where the determination of scientific thought is shown to be historical and social as well as cognitive. The mystery is solved by realizing that cognition takes place in a cultural environment, which is historically constructed. ${ }^{5}$ One should therefore combine sociocultural explanations with cognitive ones. It is one of the main goals of this volume to explore how these explanations could or should be combined,

\footnotetext{
${ }^{5}$ More controversially, I would view cognitive anthropology as providing the empirical expression of social externalism, which in turn solves the deadlocks of internalism.
} 
and one of the results is an emphasis on culturally situated and embodied cognition.

Reciprocally, the Sociology of Scientific Knowledge has often been accused of forgetting the role of the world on the formation of scientific knowledge (e.g. Sokal, 1998). The solution of Latour and Woolgar is to conflate the world with our representation of it (Latour, 1987; Woolgar, 1989). For this reason, and although Latour has welcomed the developments of distributed cognition (Latour, 1996) and many similarities can indeed be seen between Actor Network Theory and Distributed Cognition (Giere \& Moffatt, 2003; Giere, this volume) the approaches still remain radically incompatible. The first incompatibility lies in the fact that cognitive agents of distributed cognitive systems, and in particular human cognitive agents, need to be analysed: they perform their own cognitive tasks for the system, involving cognitive processes that are not to be disregarded. In other words, and contra Latour and Woolgar, cognitive psychology is highly relevant to science studies. The second incompatibility, maybe the most important, lies in the fact that distributed cognitive systems are partly, but essentially, defined through their input/output. For the distributed cognitive systems of science the input is provided by the part of the world or phenomena under investigation and the output consists of the scientific knowledge generated by the system. The task of the science studies student using this framework is to account for the output by describing the cognitive processes of the system operating on the input. So microbes, for instance, are not part of the cognitive system that allowed 'the pasteurization of France' (Latour, 1993), they just provide the input. The sociology of scientific knowledge from the Edinburgh School, on the other hand, has offered no metaphysical twist for answering the accusation: it has simply stated that there are "other types of causes apart from social ones which [...] cooperate in bringing about belief" (Bloor, 1991: 7). These other types of causes, says Bloor, ${ }^{6}$ are to be found in the constraints and the constructive role of the mind when it processes the stimuli provided by the world. So the phenomena under scientific investigation have a causal role when they stimulate our senses, or, more generally (since scientific artefacts often mediate between the phenomena and the human senses),

\footnotetext{
${ }^{6}$ See e.g. Bloor's afterword to the second edition of Knowledge and Social Imagery (1991), Bloor (1992), Barnes, Bloor \& Henry (1996) and Bloor (1997).
} 
when they provide an input to the distributed cognitive systems of science. The main difficulty for the science studies student that has been pointed by the sociologists of science is to avoid describing the input in the same terms that are used for the output. Only with such a constraint will the science studies student be able to point out the processes undertaken by a distributed cognitive system. Thus, when Spranzi says that Galileo saw dark marks on the moon, this allows her to explain why and how these marks came to be seen as shadows of mountains. An analysis of the flow of information through cognitive analysis allows identifying the input and the output of cognitive systems: by recognising the causal role of the former in the production of the latter, one can point out where the external world (i.e. external to the cognitive system) intervenes. But of most interest for the science studies student is what happens in between the input and the output: the social and cognitive construction of scientific knowledge.

What remains of the impossibility of an integrated, naturalised, investigation of science? In principle, nothing. In practice, nothing. Cognitive anthropology provides the science studies student both with the conceptual tools I have mentioned above, but also with the methods of investigation.

\section{A word on methods}

Science studies - because it is an interdisciplinary field - has numerous methods of investigation at its disposal. Klahr and Simon (1999) distinguish historical studies, laboratory studies, direct observation and computational modelling, ${ }^{7}$ and show how they complement each other. The main method of cognitive anthropology of science is direct observation, which is what anthropologists have long called participant observation: the observation of

\footnotetext{
${ }^{7}$ Dunbar and Fugelsand (in press) add the study of brain patterns using techniques such as magnetic resonance imaging and use different names: they distinguish in vitro (laboratory studies), ex vivo (direct observation), in silico (computational modelling), in magnetico (study of brain patterns), sub specie historiae (historical studies). I would add that, with the development of evolutionary psychology, specie historiae is made highly relevant: see Tooby and Cosmides (1992) for a general argument on the relevance of evolutionary psychology for the study of culture; Carruthers (2002) for an argument regarding the specific skills for tracking that evolved for hunting and gathering and which would sustain scientific cognition; evolutionary argument could also increase the plausibility of Atran's hypothesis of the existence of a module for reasoning with life kinds.
} 
scientific practices in real life settings by being present in these settings and participating in the research activities (e.g. Latour \& Woolgar, 1986).

Dunbar (1995) notes however that anthropological studies of this kind are not normally concerned with the cognitive processes that are used by scientists in their day-to-day research. He thus successfully introduced the term in vivo studies for ethnographic investigations focusing on scientific cognition and showed its fruitfulness, leading to findings complementary to laboratory, in vitro, studies (e.g. Dunbar, 1995; Dunbar \& Blanchette, 2001). For instance, while in vivo studies allow discovering the causal role of the context in the generation of analogy, in vitro studies allow further identification of which aspects of the context have a causal role.

In parallel, Hutchins has advocated a method of investigation cognitive ethnography - that is adapted for the analysis of cognition in the wild, as embodied, culturally immersed and socially distributed. Cognitive ethnography describes what are the cognitive tasks of a system and its elements, focuses on events, and, most importantly, brings together relevant techniques for achieving its goal, including interviews, surveys, participant observation and a special attention to video and audio recording. So the difference between in vivo study and cognitive ethnography, if any, is that the former emphasises the reasoning practices of scientists in research situations while the latter privileges the description of external cognitive processes such as the manipulation and transformation of external representations.

Yet another method of investigation can be associated with cognitive anthropology of science: It is cognitive history of science as defined by Nersessian (1995) and insofar, of course, as it takes the historical cultural setting into account. By incorporating historical anthropology, cognitive anthropology of science takes as material of investigation the (highly valuable) past scientific cultures; and by using a cognitive historical approach, it focuses on science as processing and producing representations. A further point renders cognitive history essential for cognitive anthropology of science: the latter should aim to account for the development of science such as conceptual change. Thus cognitive anthropology of science cannot neglect the historical approach (Kurz-Milcke et al., this volume).

The papers of this volume illustrate, first, the importance of participant observation (esp. Roth; Kurz-Milcke et al.), specifically in vivo 
studies (esp. Gooding) and specifically cognitive ethnography (esp. Alac \& Hutchins), and second, the range of techniques used: cognitive history (esp. Spranzi; Tweney), in vitro studies and statistical analysis for the investigation of the cultural bias (Poling \& Evans) and think aloud protocols (Roth). Eventually, Tweney blurs the boundaries of methods of investigation by showing how replications of past experiment allow in vitro cognitive historical studies! $!^{8}$ Two other contributions to the reflection on methodology are made in this volume: Pointing out the richness of the practice of mathematics (but the argument would certainly hold for empirical sciences) Van Bendegem \& Van Kerchove provide analytical concepts for selectively dealing with the mass of information that ethnography provides, through thematically oriented axes of research. Giere, for his part, while welcoming and contributing to the analysis of scientific distributed cognitive systems, warns us against misleading analogies between the mental and the social organisation of scientific knowledge production.

\section{Overview of the volume}

This special issue contains eleven articles ranging from detailed case studies, to methodological papers. These papers focus on four themes - Cognitive processes in scientific cultures, visual cognition and scientific practice, the cognitive structure of scientific research and methodology study. ${ }^{9}$

Cognitive processes in scientific cultures

Starting from a historical account and critical analysis of the science/ethnoscience distinction, Roy Ellen, discusses the traits that can be taken as characterising scientific cognition. Marta Spranzi's paper is a cognitive historical study of the reasoning that lead Galileo to discover the

\footnotetext{
${ }^{8}$ Another research method that blurs such boundaries is computational modelling of social phenomena. I hold that it is possible to model scientific distributed cognitive system through Distributed Artificial Intelligence. To my knowledge, however, this technique is not yet exploited. But see Thagard (1993) for a rather pessimistic view and my own ongoing research on this topic, available at http://web.epistemology.free.fr/

${ }^{9}$ This ad hoc ordering is of course limited and the all papers are relevant to more than one category. I hope I have given an idea of this by associating the papers with other themes in the previous section.
} 
mountains of the moon. Spranzi argues that Galileo's analogy is based on model construction, through bootstrapping processes.

Deveraux Poling \& E. Margaret Evans analyse ecological reasoning and its relation to intuitive beliefs, religious beliefs and scientific expertise. They show that fundamentalist American religious beliefs (esp. creationism) impede reasoning in accord with current neo-darwinian theories, but assert that, rather than changing their worldview, people would select specialisations compatible with it.

Jean Paul Van Bendegem \& Bart Van Kerkhove point out the richness of mathematical practice and analyze its constituent parts. They thus provide an analytical framework for developing complementary perspectives in historical investigations.

Visual cognition and scientific practice

The following three papers investigate the principles of scientific visual cognition.

Based on two case studies, David Gooding shows how visual images are constructed through (socially distributed, cultural) cognitive processes in order to satisfy a cognitive task or a social goal.

Wolff-Michael Roth, investigates visual cognition as applied to the interpretation and construction of graphs. He shows that graph skills are strongly related to knowledge of the phenomena represented as well as familiarity with the research practices underlying the generation of graphs.

Morana Alac \& Ed Hutchins explore how computer generated images of the brain (through fMRI) are given meaning. They especially show how this process relies on cognition in action, i.e. the production, transformation and communication of representations through gestures.

\section{Cognitive structure of scientific research}

The distributed character of scientific cognition is then investigated more generally as being evolving and structured.

Elke Kurz-Milke, Nancy Nersessian \& Wendy Newstetter analyze simulative model-based reasoning as action at and in conjuction with the bench. They describe the research done in the laboratories where they conducted fieldwork as evolving distributed cognitive systems, 
which include learning environments, historical understandings of the laboratories' agenda and activities, and multiple 'interlocking' models.

Angeles Erana \& Sergio Martinez propose a somewhat similar view of the structure of scientific knowledge. Drawing on the continuity thesis, they defend a particular analogy between science and lay cognition that allows taking into account the essential role of technology and scientific traditions.

\section{Method and methodology}

Acknowledging that the role of artefacts and action in scientific cognition could render problematic historical investigations of scientific practice. Ryan Tweney's method of replication of past experiments allows exploring all the previously hidden aspects of scientific practice. R. Tweney recounts his own replication of Faraday's experiments with gold films and points out his own findings using the method of replication.

Ronald Giere raises questions concerning the conceptual framework of Distributed Cognition. He argues that it is neither useful nor justified to attribute agency to distributed cognitive systems, thus circumscribing our understanding of cognitive distributed systems and their usefulness as a theoretical framework.

\section{Concluding remarks}

At the heart of the work of this volume is the intention of accounting for both cognitive and contextual factors in the production of scientific knowledge. An outcome is that scientific cognition appears to be inseparable from context. Cultural representations, social interactions, and the technology of the time and its artefacts fully participate in scientific knowledge production. Thus, the authors of the volume show that scientific cognition largely relies on non linguistic representations, mental or public, such as images, graphs or simulative models. Yet, the authors have kept an open eye on the findings of cognitive psychology and work on visual cognition, mental models or cognitive heuristics have been drawn upon. In the end, the project of a cognitive anthropology of science requires gathering and unifying a wide pool of theoretical resources and methodological means. The consequent syncretism is well illustrated by E. Kurz-Milcke, N. Nersessian and W. Newstetter who label their approach 'cognitive-historically situated 
ethnography.' However, beyond the diversity of theoretical and methodological resources put to work, one can see ideas converging: compare for instance the notions of fabric of interlocking models (Kurz-Milcke et al.), heuristic structures (Erana \& Martinez) and multifaceted scientific practice (Van Bendegem \& Van Kerkhove). But more work needs to be done before some unifying framework theory arises from the cognitive anthropology of science.

\section{REFERENGES}

Barkow, J., Leda Cosmides \& John Tooby (Eds.)

1992 The Adapted Mind: Evolutionary Psychology and the Generation of Culture. Oxford: OUP. BERLIN, B. \& P. KAY

1969 Basic Color Terms: their universality and growth. Berkeley: California University Press. BLOOR, D.

1991 [1976] Knowledge and Social Imagery, 2nd ed. Chicago: University of Chicago Press.

1992 'Ordinary Human Inferences as Material for the Sociology of Knowledge.' Social Studies of Science 22(1): 129-139.

1997 'Remember the strong programme?' Science, Technology, \&' Human Values 22(3): 373-385.

Carey, S. \& E. Spelke

1994 'Domain-specific Knowledge and Conceptual Change.' In L.A. Hirschfeld \& S.A. Gelman (Eds.), Mapping the Mind: Domain Specificity in Cognition and Culture Cambridge: Cambridge University Press.

Carruthers, P., S. Stich \& M. Siegal

2002 'Introduction: What Makes Science Possible?' In P. Carruthers, S. Stich \& M Siegal (Eds.), The Cognitive Basis of Science, Cambridge: CUP.

Ghurchland, P.

1988 'Perceptual Plasticity and Theoretical Neutrality: A Reply to Jerry Fodor.' Philosophy of Science 55(2): 167-187.

DUNBAR, K.

1995 'How scientists really reason: Scientific reasoning in real-world laboratories.' In R.J. Sternberg \& J. Davidson (Eds.), Mechanisms of insight. Cambridge MA: MIT press, pp. 365-395.

Dunbar, K. \& I. BlanchetTe

2001 'The Invivo/invitro Approach to Cognition: the Case of Analogy.' Trends in Cognitive Sciences 5: 334-339.

Dunbar, K. \& J.A. Fugelsang

In Press 'Causal Thinking in Science: How Scientists and Students Interpret the Unexpected.' In M. Gorman, R. Tweney, D. Gooding \& A. Kincannon (Eds.), New Directions in Studies of Scientific and Technological Thinking. Mahwah, NJ Lawrence Elbraum. 
FODOR, J.

1983 The Modularity of Mind. Cambridge, MA: MIT Press.

Fuller, S., M. De Mey, T. Shinn \& S. WoOlgar (EDS.)

1989 The Cognitive Turn: Sociological and Psychological Perspectives on Science. Dordrecht, The Netherlands: Kluwer Academic Publishers.

FREEDMAN, E.

1997 'Understanding Scientific Discourse: A Strong Programme for the Cognitive Psychology of Science.' Theory and Review ???.

Giere, R. \& B. Moffatt

2003 'Distributed Cognition: Where the Cognitive and the Social Merge.' Social Studies of Science 33 (April 2003): 301-310.

GIERE, R.

1988 Explaining Science: A Cognitive Approach. Chicago, IL \& London: The University of Chicago Press.

1994 'The Cognitive Structure of Scientific Theories.' Philosophy of Science 61(2): 276296.

GOLDMAN, A.

1986 Epistemology and Cognition. Cambridge, MA: Harvard University Press.

1994 'Naturalistic epistemology and reliabilism.' In French, Uejling \& Wettstein (Eds.), Philosophical Naturalism, pp. 301-320.

HACKING, I.

1999 The Social Construction of What? Cambridge: Harvard University Press.

HirsGhFelD, L.A. \& S.A. GelmaN

1994 Mapping the Mind: Domain Specificity in Cognition and Culture. Cambridge: Cambridge University Press.

JOHNSON-LAIRD, P.N.

1983 Mental Models. Cambridge, MA: Harvard University Press.

KLAHR, D. \& H.A. SIMON

1999 'Studies of Scientific Discovery: Complementary Approaches and Convergent Findings.' Psychological Bulletin 125(5): 524-543.

LATOUR, B. \& S. WOOLGAR

1986 Laboratory Life: The Construction of Scientific Facts. Princeton, NJ: Princeton University Press.

LATOUR, B.

1987 Science in Action. Cambridge, MA: Harvard University Press.

1993 The Pasteurization of France. Cambridge, MA: Harvard University Press.

1996 'Review of Ed Hutchins' Cognition in the Wild.' Mind, Culture and Activity 3(1): 54-63.

LONGINO, H.E.

2002 The Fate of Knowledge. Princeton, NJ: Princeton University Press.

Nersessian, N.

In Press 'Interpreting Scientific and Engineering Practices: Integrating the cognitive, social, and cultural dimensions.' In M. Gorman, R. Tweney, D. Gooding \& 
A. Kincannon (Eds.), Nerw Directions in Studies of Scientific and Technological Thinking. Mahwah, NJ: Lawrence Elbraum.

1995 'Opening the Black Box: Cognitive Science and History of Science.'

1992 In the theoretician's laboratory: Thought experimenting as mental modeling. PSA 2, 291-301.

L. Magnani and N.J. Nersessian (EDs.)

2002 Model-Based Reasoning: Science, Technology, Values. New York: Kluwer Academic/Plenum Publishers.

Pickering, A.

1991 'Philosophy Naturalized a Bit.' Social Studies of Science 21(3): 575-584.

QUINN, N.

1987 'Convergent Evidence for a Cultural Model of American Marriage.' In D. Holland and N. Quinn (Eds.), Cultural Models in Language and Thought. Cambridge: Cambridge University Press.

SLEZAK, P.

1989 'Scientific discovery by computer as empirical refutation of the strong programm.' Social Studies of Science 19: 563-600.

SPERBER, D.

1996 Explaining Culture: A Naturalistic approach. Oxford: Blackwell.

SPERBER, D. \& L.A. HirsGhFELD

2004 'The cognitive foundations of cultural stability and diversity.' Trends in Cognitive Science 8(1): 40-46.

Thagard, P.

1993 'Societies of minds: Science as distributed computing.' Studies in History and Philosophy of Science 24: 49-67.

Tooby, J. \& L. Cosmides

1992 'The Psychological Foundations of Culture.' In J. Barkow, Leda Cosmides \& John Tooby (Eds.), The Adapted Mind: Evolutionary Psychology and the Generation of Culture. Oxford: OUP, 1992.

WOOLGAR, S.

1987 'Representation, Cognition and Self: What Hope for an Integration of Psychology and Sociology.' In S. Fuller, M. De Mey, T. Shinn \& S. Woolgar (Eds.), The Cognitive Turn: Sociological and Psychological Perspectives on Science. Dordrecht, The Netherlands: Kluwer Academic Publishers, pp. 201-225. 